\title{
ARTICLE
}

\section{The effect of concrete shielding on neutron transport in the VVER-1000 mock-up}

\author{
Michal Košt'ál ${ }^{a^{*}}$, Ján Milčák ${ }^{\mathrm{a}, \mathrm{b}}$, Vojtěch Rypar ${ }^{\mathrm{a}}$, Vlastimil Juř́iček ${ }^{\mathrm{a}}$, Evžen Novák ${ }^{\mathrm{a}}$, Antonín Kolros ${ }^{\mathrm{b}}$, \\ František Cvachovec $^{c}$ and Sergey Zaritski ${ }^{\mathrm{d}}$ \\ ${ }^{a}$ Research Center Rez Ltd., 25068 Husinec-Rez 130, Czech Republic; ${ }^{b}$ CTU in Prague, Faculty of Nuclear Sciences and Physical \\ Engineering, Department of Nuclear Reactors, V Holešovičkách 2, 18000 Prague 8, Czech Republic; ${ }^{c}$ University of Defence, \\ Kounicova 65, 66210 Brno, Czech Republic; ${ }^{d}$ RRC Kurchatov Institute, 1 Kurchatov Sq., Moscow, 123182, Russia
}

\begin{abstract}
The paper shows a comparison of measured and calculated parameters of fast and thermal neutron transport in the reactor pressure vessel mock-up and in the model of biological shielding of the VVER-1000 reactor, which are placed in the LR-0 experimental reactor. The effect of concrete shielding on the transport of thermal neutrons in the surrounding area was also investigated. Transport calculations were performed by MCNP code using CENDL 3.1 and ENDF/B VII data libraries.
\end{abstract}

Keywords: VVER-1000; LR-0; MCNPX; ${ }^{3}$ He reaction rates; neutron spectroscopy; thermal scatter law

\section{Introduction}

The thermal neutron distribution in the VVER 1000 mock-up is significantly different from the fast neutron distribution. The reason for this is the concrete as the construction material of the shielding model which significantly changes the thermal neutron flux in its vicinity.

The estimation of concrete and or steel simulator effects on thermal neutron flux was done by means of measuring thermal neutron fluxes in the RPV simulator, when the reactor vessel or concrete shielding were covered with a cadmium sheet, which absorbs thermal neutrons.

When the reactor vessel had been covered, no thermal neutrons from lateral reflector entered the RPV simulator and thermal reactions on ${ }^{3} \mathrm{He}$ were mainly formed by albedo on iron and neutrons transmitted by the cadmium filter (covering 0.3-0.8 eV region). The calculation estimates that transmitted neutrons make about $20 \%$ of the total reaction rate.

Calculations were performed with MCNP code [1] using both ENDF/B VII [2] and CENDL 3.1 [3] libraries.

\section{Reactor arrangement}

The experiments were done on the LR-0 reactor, a light water zero-power research reactor operated by Research Center Rez, Czech Republic. The core of the VVER-1000 mock-up for transport benchmark used in

\footnotetext{
*Corresponding author. Email: kostal@cvrez.cz
}

these experiments consists of 32 shortened VVER-1000 type fuel assemblies with different enrichments of ${ }^{235} \mathrm{U}$ [4], [5]. The general layout of the core, VVER-1000 internal structure including the reactor pressure vessel and the concrete shielding models, is in Figure 1.

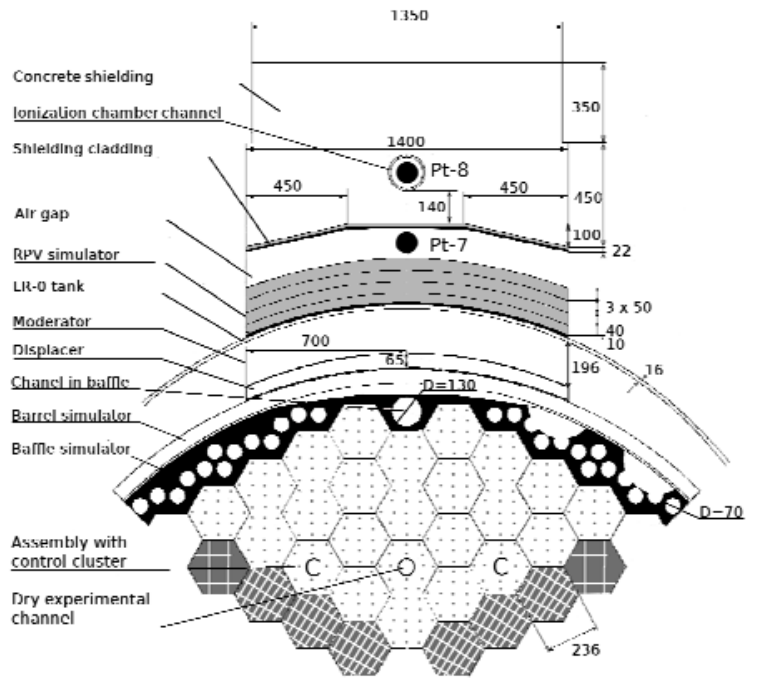

Figure 1. LR-0 Mock-up arranged for measurement at point Pt-7.

Demineralized water with diluted boric acid of the concentration of $4.6 \pm 0.1 \mathrm{~g} / \mathrm{kg}$ is used as moderator. Its depth is $150 \mathrm{~cm}$, thus the end of the fuel fissile column is $25 \mathrm{~cm}$ below the moderator level. This upper reflector is necessary for decreasing of the albedo. The criticality in this arrangement is adjusted by 2 control clusters ( 3 absorbing $\mathrm{B}_{4} \mathrm{C}$ rods only, in the position marked with a "C") raised to $60.4 \mathrm{~cm}$ above the bottom of the fissile 
column. The displacer simulates the downcomer water density reduction which takes place in the VVER-1000 power reactor at operational conditions. Various thicknesses of the VVER-1000 pressure vessel simulator can be achieved due to its modular construction. It consists of four $5 \mathrm{~cm}$ thick steel blocks. The last 3 are compact, while the nearest to the LR-0 vessel is assembled from two attached steel sectors, $1.0 \mathrm{~cm}$ layers of stainless steel (simulating RPV cladding) and $4.0 \mathrm{~cm}$ of steel layer. Measurement in points Pt-3 (before RPV), Pt-4 (RPV 1/4 thickness), Pt-5 (RPV 1/2 thickness), Pt-6 (RPV $3 / 4$ thickness), Pt-7 (RPV outer surface) and Pt-8 (tube in shielding model) were realized and analyzed.

\section{Experimental arrangement}

The fast neutron spectra in the energy range from 0.5 to $10 \mathrm{MeV}$ were measured by the proton-recoil method using a stilbene scintillator with neutron and gamma pulse shape discrimination. The spectrometric system is described in [6].

The thermal neutron flux was measured by ${ }^{3} \mathrm{He}$ proportional counter $6 \mathrm{NH} 12.5$ Canberra (active diameter $9 \mathrm{~mm}$, active length $125 \mathrm{~mm},{ }^{3} \mathrm{He}$ pressure $8 \mathrm{bar}$ ). The signal was processed by EMK-310, a high performance, portable, three-channel fast pulse-height analyzer designed for counting applications (more details can be found in [7]. Due to the pulse-mode measurement, a correction of dead time was applied.

\section{Fast neutron transport}

In case of fast neutron transport behind the LR-0 reactor vessel, through the RPV simulator and concrete shielding model, flux density considerably decreased. Figure 2 shows a comparison of calculated (using ENDF VII) and measured neutron flux densities. The calculation was normalized to the experimental value to equalize the integrals over $1 \mathrm{MeV}$ at Point Pt-3. A relatively good agreement of the experiment and the calculation was achieved with the exception of the region between 2 and $3 \mathrm{MeV}$. This discrepancy reflects the low resolution of stilbene detector, which does not allow differentiation of spectrum peak due to negative resonances of ${ }^{16} \mathrm{O}$ isotope at the energy of $2.35 \mathrm{MeV}$. A similar discrepancy can be observed in fast neutron transport in water [8]. The discrepancy in concrete is greater than those in water layer of an equivalent thickness, because the concrete has roughly six times more oxygen atoms per a hydrogen atom than the water. Apart from spectrum decreasing, a modification of the spectrum by a material through which neutrons pass is also apparent. A spectrum softening, which is an increase in the low energy part of the spectrum, during transport through the RPV model can be observed. It is caused by the cross section of the steel which forms the RPV simulator. In the concrete, the opposite phenomenon is observed, similar to the transport through water [8], i.e. a hardening of the spectrum with an increasing number of higher energy neutrons. This similarity is caused by the fact that most interactions (about $75 \%$ ) are with oxygen or hydrogen nuclei. A remarkably good agreement of the experiment with the calculation was found in the spectral index in the concrete, when a better agreement was reached using data from the library ENDF/B VII than from the CENDL 3.1 library.

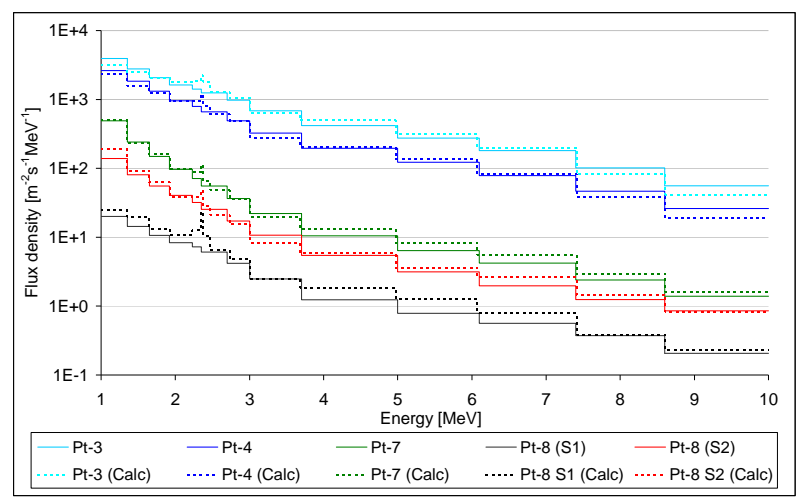

Figure 2. Comparison of calculated and measured neutron spectrum above $1 \mathrm{MeV}$.

Table 1. Spectral indices in the RPV and concrete shielding.

\begin{tabular}{lcccc} 
& Measured & \multicolumn{2}{c}{ C/E-1 } & Unc. \\
& & ENDF & CENDL \\
& & VII & 3.1 \\
Pt-3 & 3.37 & $-6.1 \%$ & $-6.7 \%$ & $4.4 \%$ \\
Pt-4 & 4.21 & $-3.5 \%$ & $-5.9 \%$ & $4.4 \%$ \\
Pt-7 & 8.60 & $-10.3 \%$ & $-14.2 \%$ & $5.9 \%$ \\
Pt-8 S1 & 4.70 & $-0.6 \%$ & $-10.0 \%$ & $4.2 \%$ \\
Pt-8 S2 & 6.07 & $12.5 \%$ & $5.4 \%$ & $3.9 \%$
\end{tabular}

For fast neutron flux density attenuation coefficients (see Table 1), a good agreement for the transport through the RPV was achieved; the discrepancies appear after the transmission through the last RPV model layer (marked as 6/7). A significantly higher discrepancy occurs especially for the attenuation in concrete shielding. The experiment differs from the calculation by up to $20 \%$. The reasons for this difference could be found in inaccuracies of the material and geometrical descriptions or of the used nuclear data. To distinguish a possible effect of concrete material, measurements were made in another arrangement of the same dimensions, but with the collimator filled with air (see Figure 3).

The calculation of flux attenuation between points Pt-7 and Pt-8 (S2) over $1 \mathrm{MeV}$ does not differ by more than $8 \%$ from the experiment. This attenuation is due to the distance and steel thickness $(2.2 \mathrm{~cm}$ simulator of concrete shielding cladding). In case of thickness effects of the concrete (marked as "S2 / S1") C/E-1 ratio reaches $15 \%$ to $20 \%$. Based on this result we can say that in energy region above $1 \mathrm{MeV}$ a greater part of the uncertainty is associated with the material rather than a geometric description.

The uncertainty, shown in the middle two rows of Table 2, is combined experimental and calculational 
uncertainty. Calculational uncertainty includes parameter uncertainty and statistical uncertainty of MCNP calculation, which does not exceed 2\% thus convergence requirements are met. Parameter uncertainty includes uncertainty in components with the greatest influence as water content and concrete density. To estimate the impact of data, calculations in library CENDL 3.1 were performed. These calculations differ slightly from the results obtained with ENDF/B VII library. The summary of the results is in Table 2.
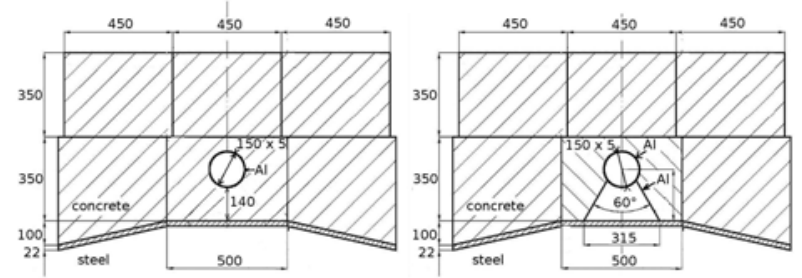

Figure 3. Concrete arrangements S1 and S2.

Table 2. Attenuation parameters of the RPV and concrete shielding

\begin{tabular}{cccccc} 
& $3 / 4$ & $4 / 7$ & $7 / \mathrm{S} 2$ & $\mathrm{~S} 2 / \mathrm{S} 1$ & $7 / \mathrm{S} 1$ \\
& \multicolumn{5}{c}{ Measured } \\
$>1 \mathrm{MeV}$ & 1.74 & 8.55 & 2.82 & 5.31 & 14.96 \\
$>3,01 \mathrm{MeV}$ & 2.18 & 17.45 & 1.99 & 4.11 & 8.18 \\
\multicolumn{5}{c}{ C/E-1 ENDF/B VII } \\
$>1 \mathrm{MeV}$ & $5.4 \%$ & $-8.8 \%$ & $-10.9 \%$ & $-11.0 \%$ & $-20.8 \%$ \\
$>3,01 \mathrm{MeV}$ & $8.4 \%$ & $-15.3 \%$ & $11.7 \%$ & $-21.4 \%$ & $-12.2 \%$ \\
\multicolumn{7}{c}{ C/E-1 CENDL 3.1 } \\
$>1 \mathrm{MeV}$ & $4.5 \%$ & $-4.1 \%$ & $-10.1 \%$ & $-9.0 \%$ & $-18.1 \%$ \\
$>3,01 \mathrm{MeV}$ & $5.4 \%$ & $-12.6 \%$ & $10.4 \%$ & $-22.2 \%$ & $-14.1 \%$ \\
$>1 \mathrm{MeV}$ & $5.5 \%$ & $6.3 \%$ & $8.4 \%$ & $7.8 \%$ & $6.5 \%$ \\
$>3,01 \mathrm{MeV}$ & $5.7 \%$ & $6.2 \%$ & $7.7 \%$ & $7.3 \%$ & $6.2 \%$
\end{tabular}

\section{Thermal neutron transport}

The situation of thermal neutron transport at experimental points behind LR-0 reactor vessel is significantly different than in the case of fast neutron transport. The comparison of calculated neutron spectra in the whole energy range at selected points in the mock-up is shown in Figure 4.

Perhaps the most notable difference is the fact that thermal neutron flux density, unlike fast neutron flux density, does not have a decreasing character.

This relates to the fact that the formation of thermal neutrons strongly depends on the environment and that the conditions are the best in a material with light nuclei and low absorption. For this reason, the thermal flux follows a strong decrease in the RPV steel mock-up and, on the contrary, an increase in the vicinity of the concrete shielding, whose substantial part is hydrogen.

A significant difference, compared with the fast neutron transport, is a greater discrepancy between the experimentally and computationally established

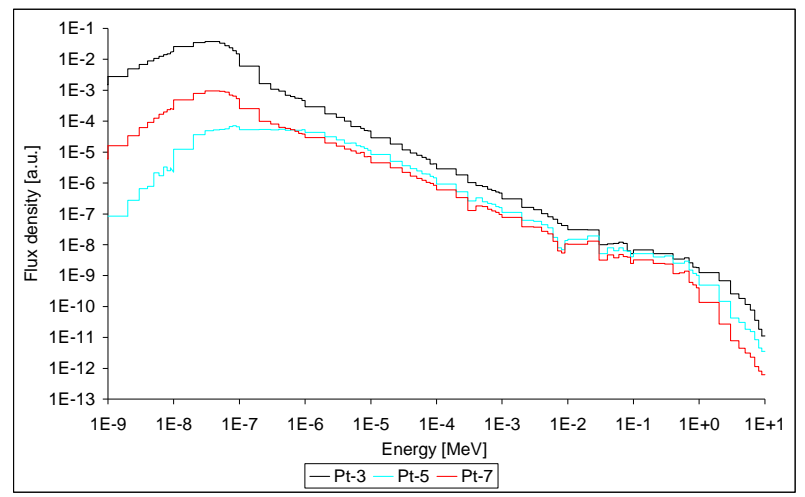

Figure 4. Calculated neutron flux densities in VVER-1000 Mock-Up from 1E-9 to $10 \mathrm{MeV}$, ENDF VII

attenuation coefficients for thermal neutron induced ${ }^{3} \mathrm{He}$ reaction rates. A slightly better agreement was reached for ${ }^{3} \mathrm{He}$ reaction rate on the epithermal neutron attenuation, which were separated from the thermal one with a $\mathrm{Cd}$ filter. The reason could lie in possible discrepancies in the description of thermal neutron angular scattering. Because of a strong attenuation, where the backscattered or reflected neutrons are involved in the response to a lesser degree, the differences between the calculation and the experiment are not too large. Using thermal scattering data for iron [2], a slightly better agreement for deeper-lying layers can be seen, but for the first layer there is a significant inconsistency in attenuation. As expected, there are no great differences between calculated results of attenuation coefficients in the epithermal reaction rate obtained using free gas model and thermal scattering matrix. The reason is that thermal scattering affects particularly thermal transport in the thermal region, while its influence above $0.5 \mathrm{eV}$ is small. The results are listed in Table 3.

Table 3. Attenuation coefficients of ${ }^{3} \mathrm{He}$ reaction rates.

$\begin{array}{ccccccc} & 3 / 4 & 4 / 5 & 5 / 6 & 6 / 7 & 3 / 7 & 7 / \mathrm{S} 1 \\ \text { Thermal } & 18.68 & 3.99 & 1.23 & 0.28 & 25.88 & 0.20 \\ \text { Epith. } & 2.54 & 1.85 & 1.42 & 1.02 & 6.77 & 3.26 \\ \text { C/E-1 ENDF/B VII } \\ \text { Thermal } & 8.9 \% & 121.1 \% & 33.7 \% & -58.9 \% & 32.1 \% & -34.1 \% \\ \text { Epith. } & 15.2 \% & 10.7 \% & 14.9 \% & -5.5 \% & 38.4 \% & -55.2 \% \\ \text { C/E-1 TSL } \\ \text { Thermal } & -59.0 \% & 39.7 \% & 76.9 \% & -1.2 \% & 0.1 \% & -22.5 \% \\ \text { Epith. } & 4.9 \% & 18.2 \% & 11.0 \% & -5.3 \% & 30.3 \% & -54.0 \% \\ \text { Chermal } & -3.9 \% & 101.8 \% & 37.2 \% & -45.4 \% & 45.4 \% & -44.2 \% \\ \text { Epith. } & 4.8 \% & 10.1 \% & 5.0 \% & -1.6 \% & 19.1 \% & -52.0 \% \\ \text { Thermal } & 18.1 \% & 12.4 \% & 11.9 \% & 11.3 \% & 17.5 \% & 4.1 \% \\ \text { Epith. } & 10.9 \% & 14.7 \% & 16.0 \% & 15.9 \% & 13.1 \% & 5.2 \%\end{array}$

A very interesting fact is that for epithermal reaction rates and RPV attenuation coefficient a better agreement of the experiment and the calculation is for data from 
CENDL 3.1 library. However, the degree of inconsistency in ${ }^{3} \mathrm{He}$ reaction rates attenuation in the mock-up inner layers is similar.

The uncertainties, listed in the last row of Table 3 , are combined experimental and calculational uncertainties. The calculated uncertainties do not exceed $2 \%$, thus satisfy the MCNP statistical requirements. This condition is met also in results presented in next chapters. The experimental uncertainty ranges from about $2 \%$ to $7 \%$ in thermal reaction rates in regions with a low $\mathrm{Cd}$ ratio. When the $\mathrm{Cd}$ ratio falls to 1.19 behind reactor vessel covered with $\mathrm{Cd}$, as presented in the next chapter, the uncertainty in thermal reaction rate determination reaches $13 \%$.

The geometry (gap thickness), boron level and Mn content in the steel of RPV simulator uncertainties are covered as parameter uncertainty. It varies from $6-12 \%$.

\section{Effect of concrete shielding}

It can be assumed that behind the RPV model a majority of thermal neutrons is backscattered from biological shielding, which is made of concrete and lined by $2.2 \mathrm{~cm}$ of steel. The estimation of such effects is based on an experiment in which the Cd cover sheet was either on the LR-0 vessel measurement window or at front of the concrete shielding model, adjacent to the steel lining of the biological shielding model. In the latter case, not the entire model, but only its third was covered. The calculations show only small deviations between both arrangements ( $1 / 3$ covered vs. fully covered model). $\mathrm{Cd}$ ratio should decrease 3.13 times when the entire concrete shielding model is covered, while for $1 / 3$ coverage it should decrease 2.97 times.

When the LR-0 measurement window had been covered with a $\mathrm{Cd}$ sheet, thermal neutrons from the concrete shielding were detected together with neutrons slowed down in steel, and some part was covered by neutrons transmitted through the $\mathrm{Cd}$ (about 20\% according to the calculated estimation). The measurements of thermal neutron flux density were done using ${ }^{3} \mathrm{He}$ reaction rates. With decreasing distance from the core, the concrete shielding backscattered component would exceed the effect of slowing down in the steel. Given that $\mathrm{Cd}$ mainly affects the thermal flux, these effects could be monitored with the $\mathrm{Cd}$ ratio.

From the measured data, see Table 4, a significant decrease in Cd ratio can be observed in the first layers of the RPV simulator up to the point Pt-5 $(5 \mathrm{~cm}$ and $10 \mathrm{~cm})$, where the change reaches about twice the related uncertainties. Cd ratios with covered measurement window behind $10 \mathrm{~cm}$ of steel do not differ from the original model, suggesting that the influence of concrete shielding prevails. In comparison with the calculation, the best agreement was achieved for the measurement behind the RPV model, while the consistency at other RPV points is getting slightly worse with increasing thickness.
Table 4. Cd ratio in various arrangements of VVER-1000 Mock-Up.

\begin{tabular}{|c|c|c|c|c|c|c|}
\hline & Pt-3 & Pt-4 & Pt-5 & Pt-6 & Pt-7 & Pt-8 S1 \\
\hline \multicolumn{7}{|c|}{ Measurement } \\
\hline Mock-Up & 9.59 & 2.17 & 1.54 & 1.62 & 3.25 & 38.52 \\
\hline R.C. & 1.19 & 1.36 & 1.43 & 1.59 & 3.21 & \\
\hline C.C. & 9.42 & 2.16 & 1.51 & 1.57 & 1.61 & \\
\hline \multicolumn{7}{|c|}{ C/E-1 (ENDF/B VII) } \\
\hline Mock-Up & $8.0 \%$ & $8.2 \%$ & $-14.8 \%$ & $-19.3 \%$ & $9.8 \%$ & $-21.9 \%$ \\
\hline R.C. & $-5.5 \%$ & $-12.2 \%$ & $-16.5 \%$ & $-19.3 \%$ & $10.9 \%$ & \\
\hline C.C. & $9.1 \%$ & $5.7 \%$ & $-13.9 \%$ & $-24.1 \%$ & $-20.3 \%$ & \\
\hline \multicolumn{7}{|c|}{ C/E-1 (CENDL 3.1) } \\
\hline Mock-Up & $7.5 \%$ & $9.8 \%$ & $-12.5 \%$ & $-19.4 \%$ & $-7.7 \%$ & $-22.9 \%$ \\
\hline R.C. & $-4.8 \%$ & $-13.0 \%$ & $-18.9 \%$ & $-20.2 \%$ & $-6.2 \%$ & \\
\hline C.C. & $5.7 \%$ & $10.2 \%$ & $-11.8 \%$ & $-23.5 \%$ & $-25.3 \%$ & \\
\hline \multicolumn{7}{|c|}{ Uncertainties } \\
\hline Mock-Up & $6.7 \%$ & $7.4 \%$ & $6.5 \%$ & $7.4 \%$ & $8.6 \%$ & $5.1 \%$ \\
\hline R.C. & $4.9 \%$ & $6.2 \%$ & $6.5 \%$ & $6.7 \%$ & $8.5 \%$ & \\
\hline C.C. & $6.3 \%$ & $7.0 \%$ & $6.8 \%$ & $6.8 \%$ & $9.2 \%$ & \\
\hline
\end{tabular}

Thermal neutrons from the core were also detected together with the neutrons decelerated in the steel as well as with transmitted and multiply scattered neutrons when the concrete had a $\mathrm{Cd}$ cover. The multiply scattered part of the contribution was made by neutrons moderated in concrete shielding.

The measured data show a significant drop at Point Pt-7. At other points, although this drop was also measured, there are minor differences comparable to the uncertainties.

The worst agreement of the calculation and the experiment was reached at Point Pt-7, while the rate of non-compliance was reduced with increasing distance from the concrete. This could support the previously stated assumption of inaccuracies or problems in angular distribution of scattered neutrons, because all contributions to thermal neutron flux causing ${ }^{3} \mathrm{He}$ reactions are strongly dependent on a correct description of the angular distribution of scattered neutrons.

Given that the $\mathrm{Cd}$ ratios are directly proportional to the reaction rate in the thermal region, the changes in thermal fluxes can be observed even in them. Quantifying measured changes together with the calculated predictions are presented in Table 5.

Table 5. Change in reaction rate in various arrangements.

\begin{tabular}{|c|c|c|c|c|c|}
\hline & Pt-3 & Pt-4 & Pt-5 & Pt-6 & Pt-7 \\
\hline \multicolumn{6}{|c|}{ Measurement } \\
\hline \multicolumn{6}{|c|}{ Reactor cover } \\
\hline Thermal & $2.1 \%$ & $30.4 \%$ & $79.7 \%$ & $92.9 \%$ & $98.2 \%$ \\
\hline Epith. & $92.9 \%$ & $97.5 \%$ & $98.9 \%$ & $98.4 \%$ & $99.7 \%$ \\
\hline \multicolumn{6}{|c|}{ Concrete cover } \\
\hline Thermal & $96.1 \%$ & $97.1 \%$ & $94.2 \%$ & $87.3 \%$ & $25.0 \%$ \\
\hline Epith. & $98.0 \%$ & $98.1 \%$ & $99.9 \%$ & $95.3 \%$ & $92.0 \%$ \\
\hline
\end{tabular}

C/E-1 (ENDF VII) 


\begin{tabular}{|c|c|c|c|c|c|}
\hline Thermal & $-40.9 \%$ & $-51.6 \%$ & $-22.4 \%$ & $1.1 \%$ & $1.5 \%$ \\
\hline Epith. & $-2.2 \%$ & $3.1 \%$ & $-1.6 \%$ & $5.1 \%$ & $0.0 \%$ \\
\hline \multicolumn{6}{|c|}{ Concrete cover } \\
\hline Thermal & $2.3 \%$ & $0.6 \%$ & $0.7 \%$ & $-29.4 \%$ & $-60.4 \%$ \\
\hline Epith. & $1.2 \%$ & $4.7 \%$ & $-1.3 \%$ & $4.5 \%$ & $-2.3 \%$ \\
\hline \multicolumn{6}{|c|}{ C/E-1 (CENDL 3.1) } \\
\hline \multicolumn{6}{|c|}{ Reactor cover } \\
\hline Thermal & $-36.1 \%$ & $-56.4 \%$ & $-39.1 \%$ & $-8.5 \%$ & $0.6 \%$ \\
\hline Epith. & $-0.7 \%$ & $0.4 \%$ & $4.5 \%$ & $-0.6 \%$ & $-1.8 \%$ \\
\hline \multicolumn{6}{|c|}{ Concrete cover } \\
\hline Thermal & $3.9 \%$ & $5.4 \%$ & $1.3 \%$ & $-26.5 \%$ & $-62.0 \%$ \\
\hline Epith. & $5.9 \%$ & $4.7 \%$ & $0.6 \%$ & $3.4 \%$ & $1.2 \%$ \\
\hline \multicolumn{6}{|c|}{ Uncertainties } \\
\hline \multicolumn{6}{|c|}{ Reactor cover } \\
\hline Thermal & $17.3 \%$ & $12.2 \%$ & $11.5 \%$ & $12.8 \%$ & $12.5 \%$ \\
\hline Epith. & $10.3 \%$ & $13.9 \%$ & $16.1 \%$ & $16.7 \%$ & $16.1 \%$ \\
\hline \multicolumn{6}{|c|}{ Concrete cover } \\
\hline Thermal & $8.3 \%$ & $9.3 \%$ & $10.3 \%$ & $12.7 \%$ & $15.2 \%$ \\
\hline Epith. & $10.2 \%$ & $13.9 \%$ & $16.1 \%$ & $16.7 \%$ & $16.5 \%$ \\
\hline
\end{tabular}

\section{Conclusions}

Experiments done on the LR-0 show a significant influence of concrete shielding on transport of thermal neutrons, especially in the last quarter of the VVER-1000 pressure vessel simulator.

Thermal neutrons in the first half of the thickness of the simulator come from the moderation in the lateral reflector. A significant discrepancy in the observed parameters for the thermal neutron transport could point to inaccuracies in the description of the angular distribution of thermal neutron scattering on iron. This is supported by a strong discrepancy in case of $1 / 3 \mathrm{Cd}$ covering of the concrete shielding, where several times scattered neutrons would play a significant role, the resulting distribution would be strongly dependent on the description of scattering.

If thermal scattering matrix $S(\alpha, \beta)$ for iron is used, despite a better agreement between calculated and measured parameters in RPV simulator inner points, there is a significant discrepancy in front of or behind the RPV simulator. Very similar results were presented in [9].

Good agreement was achieved for the studied transport of fast neutrons. Higher discrepancies appear in the attenuation coefficient of the concrete shielding. Comparing models with geometrically identical concrete and empty space, it was confirmed that a significantly larger discrepancy had been caused by material parameters of concrete, rather than a geometrical parameter. A comparable agreement was reached for the calculation with ENDF VII library and CENDL 3.1 library for attenuation over $1 \mathrm{MeV}$.

Comparing the spectra in concrete shielding, a significant discrepancy can be found in the $2-3 \mathrm{MeV}$ region reflecting a large part of oxygen, whose fine structure cannot be registered in stilbene detector because of its lower resolution.

\section{Acknowledgements}

The experiments and calculations have been funded by the Research Centre Rez Ltd. within Ministry of Education, Youth and Sport Czech Republic project MSM 2672244501, by the SUSEN Project CZ.1.05/2.1.00/03.0108 realized in the framework of the European Regional Development Fund (ERDF), by the MSM 6840770040 project and University of Defense CR Specific research support [10]. The experiments have been partly performed in frame of the co-operation between Russian Research Centre "Kurchatov Institute" and Research Centre Rez Ltd.

\section{References}

[1] D.B. Pellowitz, MCNPX User's Manual, version 2.6.0, Los Alamos Report No. LA CP 02 408, (2007).

[2] ENDF/B VII.0, D00226MNYCP01, (2008).

[3] CENDL 3.1, www.nndc.bnl.gov, accesed 24.6.2010.

[4] B. Ošmera and S. Zaritsky, WWER-1000 Mock-up experiment in the LR-0 reactor, Mock-Up Description and Experimental Data, ÚJV 11815-R, RRC KI 36/27-2002.

[5] J. Kyncl, V. Rypar and E. Novak, VVER physics experiments: hexagonal lattices $(1.275 \mathrm{~cm}$ Pitch $)$ of low enriched U(3.6, 4.4 wt.\% U235)O2 fuel assemblies in light water with $\mathrm{H} 3 \mathrm{BO} 3$, LEU-COMP-THERM-086, (2008), NEA/NSC/DOC/(95)03/IV Volume IV.

[6] Z. Bures, J. Cvachovec, F. Cvachovec, P. Celeda and B. Osmera, Multiparameter multichannel analyser system for characterization of mixed neutron - gamma field in the experimental reactor LR-0 , Proceedings of the 11th Symposium on Reactor Dosimetry, Brussels, Belgium, (2002), pp. 194-201.

[7] A. Kolros, O. Huml, M. Křŕž and J. Kos, Equipment for neutron measurements at VR-1 Sparrow training reactor, Applied Radiation and Isotopes 68, Issue 4-5, April-May 2010, pp. 570-574.

[8] M. Košt'ál, F. Cvachovec, V. Rypar and V. Juříček, Calculation and measurement of neutron flux in the VVER-1000 Mock-up on the LR-0 research reactor, Annals of Nuclear Energy 40 , Issue 1, February 2012, (2012), pp. 25-34.

[9] M. Kostal, F. Cvachovec, B. Ošmera, W. Hansen and V. Juříček, Thermal scatter treatment of iron in transport of photons and neutrons, Annals of Nuclear Energy 37, Issue 10, October 2010, (2010), pp. 1290-1304.

[10] F. Cvachovec, Z. Bures and M. Komarek, Support of mathematical and physical research, Final Report of Specific Research in 2006 , University of Defence in Brno, pp. 2-6. 